\title{
A Novel Ground Metric for Optimal Transport based Chronological Age Estimation
}

\author{
Ali Akbari, Member, IEEE, Muhammad Awais, Member, IEEE, Soroush Fatemifar, Syed Safwan Khalid \\ and Josef Kittler, Life Member, IEEE
}

\begin{abstract}
Label distribution Learning (LDL) is the state-ofthe-art approach to deal with a number of real-world applications, such as chronological age estimation from a face image, where there is an inherent similarity among adjacent age labels. LDL takes into account the semantic similarity by assigning a label distribution to each instance. The well-known Kullback-Leibler (KL) divergence is the widely used loss function for the LDL framework. However, the KL divergence does not fully and effectively capture the semantic similarity among age labels, thus leading to the sub-optimal performance. In this paper, we propose a novel loss function based on optimal transport theory for the LDL-based age estimation. A ground metric function plays an important role in the optimal transport formulation. It should be carefully determined based on underlying geometric structure of the label space of the application in-hand. The label space in the age estimation problem has a specific geometric structure, i.e. closer ages have more inherent semantic relationship. Inspired by this, we devise a novel ground metric function, which enables the loss function to increase the influence of highly correlated ages; thus exploiting the semantic similarity among ages more effectively than the existing loss functions. We then use the proposed loss function, namely $\gamma$-Wasserstein loss, for training a deep neural network (DNN). This leads to a notoriously computationally expensive and non-convex optimisation problem. Following the standard methodology, we formulate the optimisation function as a convex problem and then use an efficient iterative algorithm to update the parameters of the DNN. Extensive experiments in age estimation on different benchmark datasets validate the effectiveness of the proposed method, which consistently outperforms state-of-the-art approaches.
\end{abstract}

Index Terms-Optimal Transport, Wasserstein metric, Age estimation, robust loss function, divergence measures, deep label distribution learning.

\section{INTRODUCTION}

I $\mathrm{N}$ some machine learning problems, such as age estimation, head pose estimation etc., there is an inherent semantic similarity among target classes. For instance, in the chronological age estimation problem, facial images of an individual at nearby ages are very similar due to moderate, slow and gradual ageing process. This introduces a kind of similarity or correlation among adjacent age labels (classes). By effectively utilising such semantic inter-class relationships, a learning algorithm can train a model which would generate more accurate outputs. Label distribution learning (LDL) [1] is a novel learning framework, which takes the semantic similarity among target labels into consideration. Rather than assigning a single label, adopted in traditional learning frameworks, the

The authors are with the Centre for Vision, Speech and Signal Processing (CVSSP), University of Surrey, Guildford, UK.

e-mail: \{ali.akbari,m.a.rana,s.fatemifar,sk0047,j.kittler\}@surrey.ac.uk
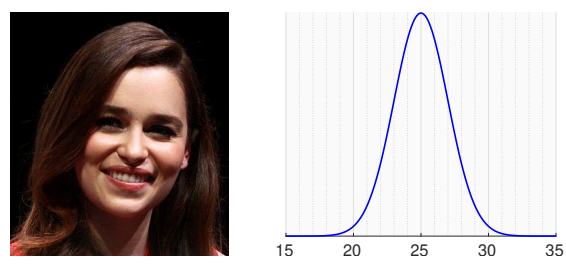

Fig. 1. Facial age estimation as a label distribution learning problem - to reflect the face similarity between neighbouring ages, the scalar age label is encoded a Gaussian label distribution for a facial image at the age of 25 .

LDL allocates descriptive degrees to all target age labels. In the age estimation problem, each degree denotes the extent to which the corresponding age label describes the instance. Obviously, the highest degree occurs at the subject's chronological age label. The complete set of all descriptive degrees constitutes a label distribution over the label space for each instance. Fig. 1 shows an instance at age 25 and its corresponding label distribution. As seen, all ages in the range 20 to 30 can describe the 25-years old face to some extent. In summary, assigning a ground-truth label distribution can implicitly utilise the semantic similarity among age labels.

The well-known Kullback-Leibler (KL) divergence [2] and the total variation (TV) distance [3] are the widely used loss functions for the LDL framework. However, these loss functions have some deficiencies, which limit their effectiveness in measuring the distance between the generated and groundtruth label distributions, resulting in sub-optimal learning performance. For instance, both the KL divergence and the TV distance return a constant value for any pair of label distributions in Fig. 2 But, intuitively, the label distribution $B$ should be closer to the label distribution $A$, as compared to the label distributions $C$ and $D$. There is also another issue associated with utilising these measures as the loss function. Although the LDL framework takes care of the semantic similarity among age labels, these measures do not fully exploit this semantic information during training. In fact, when employing the KL divergence as the loss function for training a deep neural networks (DNN), the update rule for the model's parameters in the back-propagation step ignores the relationships among age labels altogether. Clearly, in addition to the concept of label distribution encoding, the loss function itself should also reflect the semantic similarity information among labels to encourage the learning algorithm to render more accurate predictions.

One effective way of mitigating the aforementioned issues 
associated with the KL divergence and the TV distance is to exploit the optimal transport theory $[4]-[6]$. Optimal transport, also called Wasserstein distance or Earth Mover's distance as special cases, is defined as the amount of effort required to move mass to make two label distributions equal. It computes a meaningful distance between two label distributions. For instance, the optimal transport provides a smaller value for the distance between the label distributions $A$ and $B$ in Fig. 2 than for the label distributions $A$ and $C$. Leveraging this property, in this paper, we present a novel optimal transport-based loss function for the LDL framework.

Moreover, the optimal transport has another desirable benefit which helps the learning process, specifically for the age estimation problem. The optimal transport formulation adopts a ground metric function (also called transportation cost function), which captures the underlying geometric structure of the label space. This underlying geometric structure of the label space closely reflects the semantic similarity among labels. Thus in contrast to the KL divergence and the TV distance, the optimal transport based loss function exhibits appealing properties which help to capture the semantic correlation among the age labels during training.

In general, each specific learning problem will require a specific ground metric function, associated with the geometric structure of the target label space, to accurately capture the corresponding semantic similarity among labels. The existing family of optimal-transport based loss functions, called as $p$ Wasserstein [6], utilise a generic ground metric function which assumes a simple linear or quadratic structure over the label space. This may be insufficient to capture the complex (nonuniform and non-linear) similarity between close labels in some problems, such as age estimation. Motivated by this fact, in this paper, we propose a carefully devised ground metric function that incorporates the underlying label similarity structure over the age label space into the learning algorithm more effectively. The proposed loss function, named as $\gamma$-Wasserstein, is able to simultaneously address the issues associated with commonly used divergence-based loss functions and also refines the predicted label distribution so as to exert a stronger influence (cost) on errors associated with neighbouring ages.

We use the proposed loss function for training DNNs to solve the age estimation problem. This leads to a nonconvex optimisation problem, which is computationally very challenging. Our solution of this optimisation problem is inspired by the existing approaches in $[5]$ where a regularisation term is used to make the objective function convex. Starting from this regularised formulation of the transport problem, we then derive an efficient algorithm for solving the resulting optimisation problem, following the approach proposed in [6][8]. Experiments on multiple benchmark ageing datasets demonstrate substantial improvements over state-of-the-art methods.

The main contributions of this paper are summarised as follows:

- We propose an optimal transport-based loss function as an alternative to the existing loss functions.

- We handle the similarity among close ages effectively by proposing a novel ground metric function.

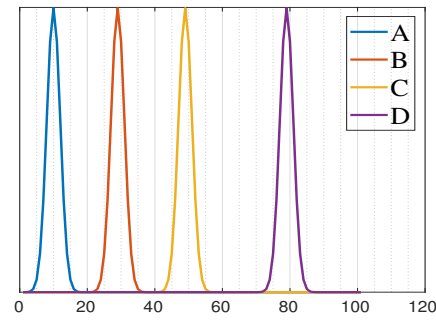

Fig. 2. The KL divergence and the TV distance are constant for all pairs of the label distributions, while the proposed $\gamma$-Wasserstein loss function outputs a distance between label distributions $A$ and $D$, which is larger than the one between label distributions $A$ and $C$ and the one between label distributions $A$ and $B$.

- To the best of our knowledge, this is the first application of optimal transport for the age estimation problem.

The rest of this paper is organised as follows: we briefly summarise the related work in Section [II] The problem formulation and the limitations of the existing loss functions, including KL divergence and TV distance, are discussed in Section III Section IV reviews the optimal transport formulation and introduces our proposed model. We provide the details of our implementation and evaluate our approach in Section V The last section concludes the paper.

\section{RELATED WORK}

In early efforts devoted to the age estimation problem, the facial appearance was represented by hand-crafted features [9] or generating a statistical model [10]. The privileged information, encoding the facial attributes of ageing, such as smoothness, face shape, face acne, wrinkles, and bags undereyes, are utilised in [11] to improve the generalisation of age estimation systems. The concepts of sparse representation [12], [13] and dictionary learning [14], [15], combined with Active Appearance Model (AAM), Local Binary Patterns (LBP), Gabor and Bio-Inspired Features (BIF), have been used in [16], [17] to extract robust face features for modelling age progression. With the popularity of DNNs in wide variety of applications [18]-[21], the research focus has shifted towards learning discriminative features by training a neural network end-to-end on a labelled face image dataset [22]. These endto-end approaches can be categorised into four major groups, namely regression, ranking, classification and label distribution based methods.

Regression based approaches are most intuitive but only a few are able to compete with other age estimation approaches [23]. It is well known that facial images possess certain ordinal patterns in the ageing feature space [24]. In the ranking based approach, this ordinality of age labels is considered during the training stage [25]-[27]. Although these approaches enhance the age estimation accuracy, they suffer from the lack of scalability. The classification based methods [28], [29] formulate the age estimation as a multi-class classification problem and treat different ages as independent classes. During the training stage, these approaches try to learn discriminative features using the well-known cross entropy (CE) loss function.

In a different approach, Geng [1] explicitly characterises the semantic correlation among age labels. In this study and 
some other similar works [2], [30], [31], each age label is represented as a label distribution (usually Gaussian or triangle label distribution). In all these methods, the KL divergence is employed as the loss function to measure the similarity between the estimated age label distribution and ground-truth. Gao et al. [31] propose the use of KL divergence regularised by an expectation regression module to further refine the value predicted by the KL loss function. The expectation regression module penalises the difference between the expectation of the estimated age label distribution and the corresponding groundtruth age label. Recently, Akbari et al. [32], [33] propose a flatter loss function for the LDL framework which exhibits a higher performance on cross-dataset settings. In this paper, we discuss that an optimal transport based loss function would exploit the underlying geometric structure of the space of label distributions more effectively as opposed to these divergencebased loss functions. Consequently, the proposed loss function is able to capture the semantic similarity among age classes more effectively.

\section{A. The Optimal Transport Theory}

Optimal transport is a metric which measures the distance between two probability label distributions using a certain ground metric function, which captures the cost of transporting mass from one label distribution to match another label distribution. Recently, the optimal transport problem has gained considerable attention in a wide range of applications, such as image classification [34], domain adaptation [35], generative adversarial networks [36], etc. There have been some efforts to integrate the optimal transport as a loss function for deep learning [7]. Most previous works employ a generic ground metric function reflecting the geometry structure in the output space to define the optimal transport problem. However, for some applications such as age estimation, the existing ground metric functions do not properly capture the semantic correlations among neighbouring labels. Different from previous works, we utilise the label structures more carefully to define a proper ground metric function.

\section{PROBlEM Formulation}

Let $\mathcal{X}$ and $\mathcal{Y}$ denote the input space and the label space over a finite set $\mathcal{L}=\left\{l_{1}, l_{2}, \cdots, l_{K}\right\}$, where $l_{k}$ is $k$-th chronological age label. The aim of an age estimation algorithm is to find a mapping function from the input space $\mathcal{X}$ to the age label space $\mathcal{Y}$. To this end, a typical method is the one-hot strategy in which a binary vector $\mathbf{y}=\left[y_{1}, \cdots, y_{K}\right]^{T} \in \mathbb{R}^{K}$ is assigned to the input instance $\mathbf{x}$ with the corresponding scalar age label $l$. If $l$ equals the $k$-th age label in $\mathcal{L}, y_{k}=1$; otherwise $y_{k}=0$. This kind of label encoding models the age estimation problem as a standard classification problem.

There is a strong visual semantic similarity among the face images of nearby ages, e.g. a person at the age 24, 25 and 26 may have very similar facial features that are difficult to distinguish. By formulating the age estimation problem as a classification problem, this semantic similarity is ignored, leading to an inconsistency during DNN training. Therefore, a network trained with the one-hot age labels has difficulty to separate visually similar face samples that have different age labels. This problem has been partially addressed by using label distribution encoding [1], by which a vector of degrees $\mathbf{y}=\left[y_{1}, \cdots, y_{K}\right]^{T} \in \mathbb{R}^{K}$ is assigned to each instance. $y_{k}$ represents the degree (probability) to which $l_{k}$ describes the instance $\mathbf{x}$. Provided that $\sum_{k=1}^{K} y_{k}=1$, the vector $\mathbf{y}$ forms a probability label distribution. So, the vector $\mathbf{y}$ is called label distribution and the learning process on instances annotated by the label distributions is called label distribution learning [37]. In this paper, we define the label distribution by a probability distribution function over $\mathcal{L}$. Following [1], [2], we use a Gaussian label distribution, where its value at the point $l_{k} \in \mathcal{L}$ is obtained via $y_{k}=\frac{1}{\sqrt{2 \pi} \sigma} \exp \left(-\frac{\left(l_{k}-l\right)^{2}}{2 \sigma^{2}}\right) ; \sigma$ is a predefined standard deviation. The highest degree of class membership is at the corresponding scalar chronological age $l$ and the probabilities gradually decrease on the both neighbouring sides of the chronological age (see Fig. 1 for reference.)

We consider the problem of learning a mapping function $h_{\theta}^{*}$, parameterized by $\theta$, over a hypothesis space $\mathcal{H}$ which maps the input instance $\mathbf{x}$ to its label distribution $\mathbf{y}$. We model this mapping function as a DNN with parameters $\theta$. Let $\hat{\mathbf{z}}$ be the DNN's output for an input image $\mathbf{x}$, i.e. $\hat{\mathbf{z}}=h^{\theta}(\mathbf{x})$. Suppose $\hat{\mathbf{z}}$ is then followed by a softmax function. The outcome of this operation is that the vector $\hat{\mathbf{z}}$ is collapsed into a probability label distribution form $\mathbf{z}=\left[z_{1}, z_{2}, \cdots, z_{K}\right] \in \mathbb{R}^{K}$, where $z_{k} \in[0,1]$ and $\sum_{k=1}^{K} z_{k}=1$. Here, $z_{k}=\exp \left(z_{k}\right) / \sum_{i} \exp \left(z_{i}\right)$ denotes the probability that sample $\mathrm{x}$ belongs to the age label $k$. Let $\mathcal{S}=\left\{\left(\mathbf{x}_{n}, \mathbf{y}_{n}\right), n=1, \cdots, N\right\}$ denote a set of training samples, where $\mathbf{x}_{n}$ and $\mathbf{y}_{n}$ represent the $n$-th training image and the corresponding ground-truth label distribution. The mapping function $h_{\theta}^{*}$ is obtained by minimising the following empirical risk:

$$
\min _{h_{\theta} \in \mathcal{H}} \frac{1}{N} \sum_{n=1}^{N} \ell\left(\mathbf{z}_{n}, \mathbf{y}_{n}\right),
$$

where $\ell(\cdot, \cdot)$ is a loss function.

The design of a proper loss function to obtain distance between pair of probability label distributions is crucial for the LDL problem. The information divergence-based measures, such as Kullback-Leibler (KL) divergence and total variation (TV) distance, are widely used as the loss function in the existing LDL algorithms [2], [37]. The KL divergence is defined as

$$
L_{K L}(\mathbf{z}, \mathbf{y})=\sum_{k=1}^{K} y_{k} \log \left(\frac{y_{k}}{z_{k}}\right),
$$

and the TV distance is defined as

$$
L_{T V}(\mathbf{z}, \mathbf{y})=\sum_{k=1}^{K}\left|z_{k}-y_{k}\right| .
$$

However, these loss functions have several deficiencies as follows.

I. Element-wise operation: As inferred from Eqs. 22 and (3), both the KL divergence and the TV distance involve a summation of $K$ element-wise operations. That means the distance between two label distributions $\mathbf{z}$ and $\mathbf{y}$ is obtained via comparing the individual bins on the label distribution 


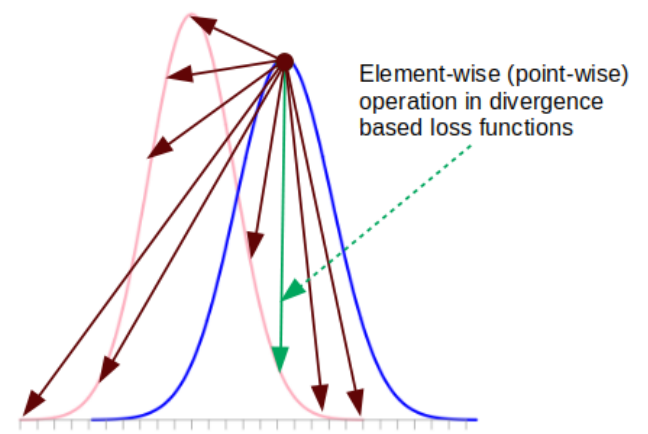

Fig. 3. Divergence-based loss function vs. OT-based loss function. The former treats the output bins independently (green line) while the latter considers the potential interaction among all the bins (brown lines).

$\mathbf{z}$, i.e. $z_{k}, k=1, \ldots, K$, with the corresponding bins on the label distribution $\mathbf{y}$, i.e. $y_{k}, k=1, \ldots, K$. In other words, these losses treat the output bins independently and thus the potential interaction among them are ignored. Fig. 3 shows the point-wise operation. In consequence, these losses do not measure the distance between the generated and ground-truth label distributions properly. Fig. 4 presents the KL divergence and the TV distance between two label distributions with their peaks at $l_{A}, l_{B}$ as a function of the label distribution separation $\Delta l=l_{A}-l_{B}$. The $\mathrm{x}$-axis plots the $\Delta l$ values and the $\mathrm{y}$-axis the corresponding loss. As seen, for small values of $\Delta l$, i.e. when the two label distributions are highly overlapping, the $\mathrm{KL}$ divergence and the TV distance assume low values. As the overlap decreases, the both loss values increase. However, as the two age label distributions become non overlapping, i.e. when $\Delta$ exceeds circa 20, the loss values saturate. The loss value reaches $\infty$ for the KL divergence, while it becomes constant for the TV distance. Hence, the KL divergence and the TV distance output a fixed distance between all the label distribution pairs in Fig. 2 Thus, once the two label distributions become disjoint, these losses ignore the actual distance between them. Consequently, the saturation of the KL and TV losses for non-overlapping label distributions may impact undesirably on the learning performance.

II. Element-wise update rule: Another issue associated with the divergence-based loss functions relates to the rule for updating the DNN's parameters during back-propagation. Using the chain rule, the derivative of the KL divergence and TV distance with respect to the model's output $\mathbf{z}$, can easily be obtained as:

$$
\partial L_{K L} / \partial z_{k}=-y_{k} \log \left(z_{k}\right)
$$

and

$$
\partial L_{T V} / \partial z_{k}=\left|z_{k}-y_{k}\right| .
$$

It is clear that during the back-propagation stage, the update of the parameters of the $k$-th element of the last layer of DNN is dependent on the difference $z_{k}-y_{k}$ of the label distributions $\mathbf{z}$ and $\mathbf{y}$. However, the update rule is oblivious of the interaction with the other bins. Ignoring the incongruence of the other bins when updating the model's parameters may render the optimisation less robust. Note that in our approach we force

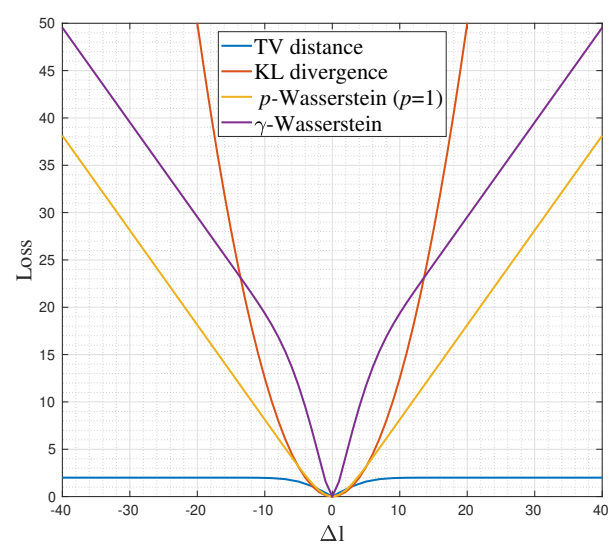

Fig. 4. The loss values produced by two label distributions with their peaks at $l_{A}, l_{B}$ as a function of age label distribution separation $\Delta l=l_{A}-l_{B}$. When both label distributions are the same, the KL divergence and the TV distance are 0 . As $\Delta l$ increases, the KL divergence and the TV distance increase. When the label distributions cease to overlap, these distances behave like a constant function. In contrast, $p$-Wasserstein and $\gamma$-Wasserstein losses continue monotonically increasing as $\Delta$ grows in magnitude.

the model to learn multi age labels with different degrees of membership via the label distribution learning framework. In fact, owing to different patterns of the ageing process, some ages (younger ages) may be easier to learn than other ages (older ages). Due to the element-wise nature of the update rule of parameters of model trained via the KL divergence and TV distance, the training process saturates too fast, and consequently results in model over-fitting on easy ages and under learning on hard ages. Intuitively, if the gradient takes into account the neighbouring ages effectively, it may redress this issue to some degree.

\section{The Methodology}

One effective way to tackle the aforementioned deficiencies associated with the KL divergence and the TV distance is to leverage the underlying geometric structure of the label space in the learning process. This can be achieved by utilising the optimal transport framework as the loss function [7]. The optimal transport [4], [6] avoids such issues by considering the pairwise cost between all the output bins when measuring the distance between pair of probability label distributions. In the following, we first describe in brief the theory of optimal transport, and the existing optimal transport based loss function, the $p$-Wasserstein loss. Then, we propose a novel optimal transport based loss function, namely $\gamma$-Wasserstein loss. The proposed loss function computes a meaningful distance between two label distributions, even when the supports of the label distributions do not overlap. Further, it facilitates an adaptive way to interact with neighbouring ages during the back-propagation stage. The proposed loss function improves the accuracy of LDL-based age estimation systems.

\section{A. Optimal Transport Theory}

Given two probability label distributions $\mathbf{z} \in \Sigma_{K}$ and $\mathbf{y} \in \Sigma_{K}$, where $\Sigma_{K}:=\left\{\mathbf{s} \in \mathbb{R}_{+}^{K} \mid \mathbf{1}^{T} \mathbf{s}=1\right\}$ is the 
$(K-1)$-dimensional probability simplex, the optimal transport (OT) problem is defined as the minimum cost of optimally redistributing the mass of one probability label distribution to match the mass of another probability label distribution, providing a measure of similarity between the two label distributions. This is formulated by optimising over transport plans that push $\mathbf{z}$ onto $\mathbf{y}$ as:

$$
\begin{aligned}
& \ell_{\mathrm{OT}}(\mathbf{z}, \mathbf{y}):=\min _{\gamma} \sum_{i=1}^{K} \sum_{j=1}^{K} g\left(l_{i}, l_{j}\right) \gamma_{i j} \\
& \text { s.t. } \quad \sum_{j=1}^{K} \gamma_{i j}=z_{i}, \sum_{i=1}^{K} \gamma_{i j}=y_{j}, \gamma_{i j} \geq 0,
\end{aligned}
$$

where $\gamma_{i j}$ represents how much mass gets moved from point $l_{i}$ to point $l_{j}$ and $g\left(l_{i}, l_{j}\right) \in \mathbb{R}^{+}$is the transportation cost of this movement. The first (second) marginal constraint in Eq. 6 satisfies that the total amount of mass transported from (to) a point $l_{i}$ must equal $z_{i}\left(y_{i}\right)$. The third constraint enforces that the amount of mass transported must be positive. It can be convenient to reformulate Eq. (6) in matrix form as:

$$
=\min _{\boldsymbol{\Gamma} \in \mathcal{U}(\mathbf{z}, \mathbf{y})}<\boldsymbol{\Gamma}, \mathbf{C}>,
$$

where $\langle\cdot\rangle$ denotes the inner Hilbert-Schmidt product for matrices. $\mathcal{U}(\mathbf{z}, \mathbf{y})$ is the set of all possible transport plans $\boldsymbol{\Gamma}=\left[\gamma_{i j}\right] \in \mathbb{R}_{+}^{K \times K}$, satisfying marginal constraints $\boldsymbol{\Gamma} \mathbf{1}=\mathbf{z}$ and $\boldsymbol{\Gamma}^{T} \mathbf{1}=\mathbf{y}$, where $\mathbf{1} \in \mathbb{R}^{K}$ is the all-one vector. $C=\left[c_{i j}=\right.$ $\left.g\left(l_{i}, l_{j}\right)\right] \in \mathbb{R}_{+}^{K \times K}$ is the cost matrix.

\section{B. The Standard p-Wasserstein Loss}

When the cost matrix $\mathbf{C}$ is obtained via a distance or ground metric function function $g: \mathcal{L} \times \mathcal{L} \rightarrow \mathbb{R}^{+}$, then it is shown in [4] that $\ell_{\text {OT }}$ satisfies the properties of a distance, referred to as optimal transport distance. In this case, the cost matrix is called ground metric matrix. The well-known $p$-Wasserstein distance [6] is defined using the OT formulation (6) with $g_{w}\left(l_{i}, l_{j}\right)=\left|l_{i}-l_{j}\right|^{p}$ for any $p \geq 1$. For the specific case of $p=$ 1 , the $p$-Wasserstein distance is also known as the earth mover distance. According to this ground metric function, the cost of optimal transport plan, obtained by $\ell_{\mathrm{OT}}$, for redistributing the predicted label distribution $\mathbf{z}=h_{\theta}(\mathbf{x})$ to match the ground-truth label distribution $\mathbf{y}$ is penalised over longer distances.

\section{The Proposed $\gamma$-Wasserstein Loss}

The ground metric function $g\left(l_{i}, l_{j}\right)$ plays an important role in building the shape of the OT-based loss function (see section IV-E for an intuitive analysis.) It is directly related to the cost needed for transporting the mass from point $l_{i}$ to point $l_{j}$. The choice of a ground metric function should reflect the geometric structure of the label space, which manifests the semantic similarity of the labels. Since the label spaces of different learning problems have different characteristics, the ground metric function should be designed to properly capture the different notions of semantic similarity among their target labels. This is particularly crucial in the age estimation problem where there are strong and complex semantic similarities among neighbouring ages.

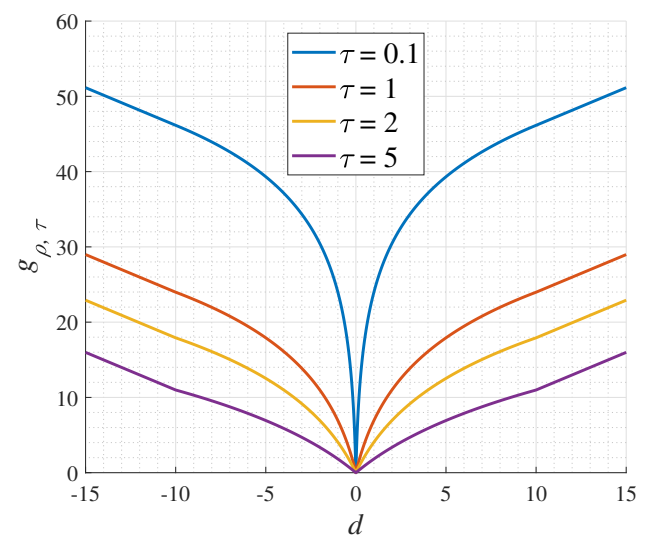

Fig. 5. The proposed ground metric function for different values of parameter $\tau(\rho=10)$.

In the optimal transport problem (7), any ground metric function in the form $g\left(l_{i}, l_{j}\right)=T\left(l_{i}-l_{j}\right)$ can be considered. Here function $T(\cdot)$ is a symmetric and monotonically increasing function with respect to $l_{i}-l_{j}$ and has a single minimum at $l_{i}=l_{j}$. Along with this direction, we formulate the ground metric function such that it reflects this general intuition, i.e. the farther the transportation, the higher the cost. More precisely, this criterion will favour the cost $g\left(l_{i}, l_{j}\right)$ to be small for pairs of close points and large for pairs of far points. In addition, we consider another constraint in designing the ground metric function: The ground metric function should emphasise the crucial importance of closer transportation. Considering these constraints, we formulate the ground metric function as:

$$
g_{\gamma}\left(l_{i}, l_{j}\right)= \begin{cases}\rho \ln \left(1+\frac{\left|l_{i}-l_{j}\right|}{\tau}\right), & \text { if }\left|l_{i}-l_{j}\right| \leq \rho \\ \left|l_{i}-l_{j}\right|-\phi, & \text { otherwise }\end{cases}
$$

where $\phi=\rho-\rho \ln (1+\rho / \tau)$ is a constant to make the metric continuous at point $\left|l_{i}-l_{j}\right|=\rho$. This metric behaves as a logarithmic function for close transfers, thus focusing on the semantic similarity between neighbouring classes more effectively than the existing ground metric functions.

The proposed ground metric function is visualised with respect to $d=l_{i}-l_{j}$ for different values of $\tau$ and $\rho=10$ in Fig. 5. The shape of the ground metric function gives some intuition about how different values of $\rho$ and $\tau$ change the behaviour of the ground metric function which may affect the optimal transport plan induced by (7). Depending on the chosen values of $\rho$ and $\tau$, a different cost is assigned to each transportation; a smaller cost to the pair of close points and vice versa. For smaller values of $\tau$, the curvature of the ground metric function is logarithmic for the close transportation. In other words, the transportation cost assigned by the ground metric function changes logarithmicly in the area where it deals with pair of close points. Depending on the value of $\rho$, the ground metric function switches to a linear section for the far point transportation. Consequently, the transportation cost assigned by the ground metric function changes linearly in this area. 


\section{Optimisation}

Given the appropriate values for $\rho$ and $\tau$, we then integrate the proposed $\gamma$-Wasserstein loss function into deep learning. For solving the risk minimisation problem (1), we first need to solve the minimisation problem (7) which is a non-convex task and with the high computational complexity $\mathcal{O}\left(n^{3} \log n\right)[38]$. Cuturi et al. [5] propose to use the regularised optimal transport (ROT) distance that speeds up the computation of the original optimal transport as:

$$
\begin{aligned}
\ell_{\mathrm{ROT}}(\mathbf{z}, \mathbf{y}) & =\min _{\gamma} \sum_{i} \sum_{j} g\left(l_{i}, l_{j}\right) \gamma_{i j}-\lambda \gamma_{i, j}\left(\ln \gamma_{i, j}-1\right) \\
& =\min _{\boldsymbol{\Gamma} \in \mathcal{U}(\mathbf{z}, \mathbf{y})}<\boldsymbol{\Gamma}, \mathbf{C}>-\lambda H(\boldsymbol{\Gamma}),
\end{aligned}
$$

Here, $H(\boldsymbol{\Gamma})$, known as Sinkhorn's relaxation, measures the complexity of the transportation plan by computing its entropy. This measure is used to regularise the hypothesis space $\mathcal{U}$ by favouring a simpler hypothesis. This entropic regularisation makes the objective function a convex problem that can be solved several times faster than the original transport problem (6). More details can be found in [5].

Utilising our proposed ground metric function for the age estimation problem, we adapt the Sinkhorn-Knopp algorithm [5], [39] to obtain the optimal solution of $\ell_{\text {ROT }}(9)$. This is achieved through performing row and column scaling (See Algorithm 1). At the next step, we need to compute the gradient of the loss function with respect to the output $\mathbf{z}$. As discussed in $[6]-[8]$, the objective function and constraints in Eq. (9) are linear with respect to $\Gamma$; therefore, it can be computed using its Lagrange duality:

$$
\begin{aligned}
d_{\ell_{\mathrm{ROT}}}(\mathbf{z}, \mathbf{y}) & =\max _{\boldsymbol{\alpha}, \boldsymbol{\beta} \in \mathbb{R}^{K}} \boldsymbol{\alpha}^{T} \mathbf{z}+\boldsymbol{\beta}^{T} \mathbf{y} \\
& -\frac{1}{\lambda} \sum_{i} \sum_{j} \lambda \exp \left(\alpha_{i}+\beta_{j}-c_{i j}\right) .
\end{aligned}
$$

The optimal solutions of the prime problem 9 and dual Lagrange problem (10) are equal and related to each other. In fact, the ROT problem (9) has a semi-closed minimiser, called optimal transport plan, $\boldsymbol{\Gamma}^{*}=\exp \left(\lambda\left(-\mathbf{C}+\boldsymbol{\alpha}^{*} \mathbf{1}^{T}+\mathbf{1} \boldsymbol{\beta}^{*}\right)\right)=$ $\operatorname{diag}\left(\mathbf{u}^{*}\right) \mathbf{T} \operatorname{diag}\left(\mathbf{v}^{*}\right)$ for $\mathbf{T}=\exp (-\lambda \mathbf{C}), \mathbf{u}^{*}=\exp \left(\lambda \boldsymbol{\alpha}^{*}\right)$ and $\mathbf{v}^{*}=\exp \left(\lambda \boldsymbol{\beta}^{*}\right)$, where $\boldsymbol{\alpha}^{*}$ and $\boldsymbol{\beta}^{*}$ are the solutions to the dual problem in Eq. $(10) . \boldsymbol{\alpha}^{*}$ and $\boldsymbol{\beta}^{*}$ can be efficiently obtained by the Sinkhorn-Knopp algorithm [7], [39]. The interested reader may find it useful to consult [5] for more details of the concepts explained. An example of the source and target label distributions, i.e. $\mathbf{y}$ and $\mathbf{z}$, and the resulting transport plan $\Gamma^{*}$ is illustrated in Fig. 6. Recall the matrix $\Gamma^{*}$ represents the possible plans of transporting the mass from the predicted output $\mathbf{z}$ to the ground-truth label distribution $\mathbf{y}$.

After solving the minimisation problem (9), we then update the DNN's weights $\theta$. Considering Eq. (10), the gradient of the proposed $\gamma$-Wasserstein loss with respect to the DNN's output, i.e. $\nabla_{\mathbf{z}} \ell_{\mathrm{ROT}}$, is computed as

$$
\nabla_{\mathbf{z}} \ell_{\mathrm{ROT}}=\boldsymbol{\alpha}^{*}=\frac{\ln \left(\mathbf{u}^{*}\right)}{\lambda} .
$$

The gradient with respect to $\mathbf{z}$ is then used to update the model's parameters $\theta$ using the chain rule. The detailed procedure is
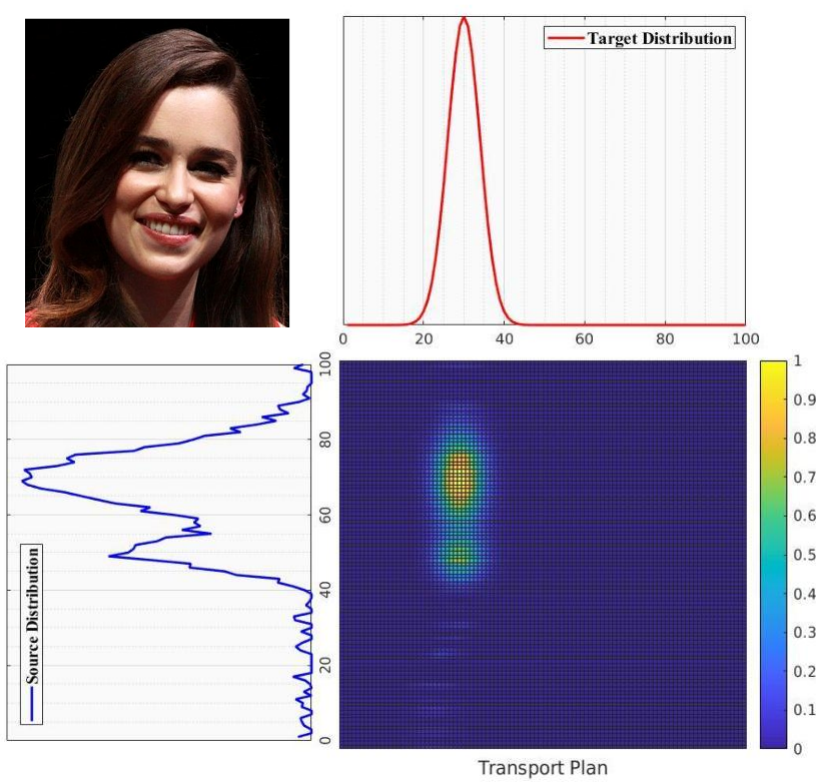

Fig. 6. Top: The target label distribution is a Gaussian for a facial image at the age of 30. Below: The source label distribution and the corresponding transport plan that maps the source label distribution onto the target label distribution (from Top). (Best viewed in colour)

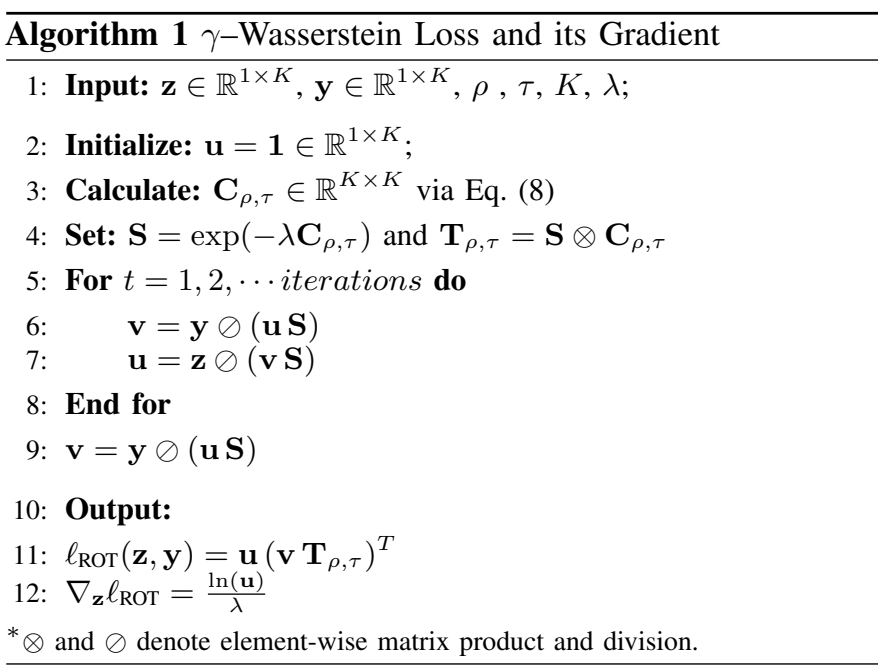

summarised in Algorithm 1. $\nabla_{\mathbf{z}} \ell_{\text {ROT }}$ is then used to update the DNN's parameters $\theta$ using the chain rule. In our experiments, we found $\rho=10, \tau=1$ and $\lambda=0.2$ to be most effective. We set the number of iterations in Algorithm 1 as 50 and the standard deviation of label distribution as $\sigma=2$ for all ages. Detailed ablation studies on parameter settings are discussed in the next section. In this study, we find the best values for $\rho$ and $\tau$ via cross validation. The detail will be given in the following sections.

\section{E. The intuitive analysis}

In this section, we provide an intuitive analysis of the properties of the proposed $\gamma$-Wasserstein loss function in comparison with the standard $p$-Wasserstein loss function 
and the divergence-based losses, i.e. KL divergence and TV distance.

I. Treatment of errors: In our proposed formulation, the ground metric has two free parameters, i.e. $\rho$ and $\tau$, which are fine-tuned during training. This allows the loss function to adaptively adjust its shape to control the influence of small errors and the impact of large errors. In following, we elaborate on how the shape of the proposed $\gamma$-Wasserstein loss effectively deals with both relatively small and large errors.

In the estimation of ages from images of faces in-thewild, we may be dealing with situations where initially the estimation error can be very large. In such cases, the loss function should facilitate a fast recovery from these large errors. Fig. 2 illustrates the proposed $\gamma$-Wasserstein loss between two label distributions as a function of the age label distribution separated by $\Delta l$. As discussed earlier, in the case of the KL divergence and the TV distance, the loss for large errors (when the two label distributions are not overlapping, see Fig. 2) is constant. Such losses are incapable of guiding the model update. As anticipated in Fig. 4, both $p$-Wasserstein and $\gamma$-Wasserstein address the saturation of the KL divergence and the TV distance for non-overlapping label distributions (large errors). These metrics behave linearly at large errors and continue monotonically increasing as $\Delta l$ grows in magnitude. Thus in contrast to the KL divergence and the TV distance, the optimal transport pays more differentiating attention to the instances with large errors. For measuring the distance between pairs of label distributions in Fig. 2, the proposed $\gamma$-Wasserstein loss respects the fact that label distribution $D$ is further from $B$ than label distribution $C$.

On the other hand, as discussed in [40], [41], loss functions emphasising small errors improve the accuracy of the DNN models. In our work, the impact of small errors is improved by incorporating the proposed ground metric function by its logarithmic form (see Fig. 5). The proposed ground metric function, the $\gamma$-Wasserstein loss, as can be seen from Fig. 4 is a concave function of $|d|$, which rapidly increases in the range of small errors. Consequently, $\gamma$-Wasserstein loss is more sensitive to small errors, compared with the $p$-Wasserstein loss function, the KL divergence and the TV distance where the loss values exhibit the quadratic growth. In general, similar to $p$-Wasserstein loss, the linear part of $\gamma$-Wasserstein loss drives the training process close to the point where predictions will be fairly close to the ground truth label distribution. Then, in contrast to the $p$-Wasserstein loss, the $\gamma$-Wasserstein loss function will switch to its logarithmic part to refine the prediction with a greater influence exerted by small errors.

II. Correlation-aware update rule: As discussed earlier, the derivative of $\gamma$-Wasserstein loss function $\ell_{\text {ROT }}$ with respect to each element of the vector $\mathbf{z}$ is obtained as $\nabla_{\mathbf{z}} \ell_{\mathrm{ROT}}=\frac{\ln (\mathbf{u})}{\lambda}$, where $\approx$ is obtained via the iterative loop in Algorithm 1. It can be inferred that the update rule of each network's parameter depends on all elements of $\mathbf{p}$ and $\mathbf{q}$ via computing matrix $\mathbf{C}$. This is more robust compared to the KL divergence and TV distance where, as discussed in Section [III the update rule depends only on the corresponding target bins, i.e. $z_{i}$ and $q_{i}$ (see Eqs. (2), (3)).

\section{EXPERIMENTS}

In this section, we first introduce the experimental settings, including the datasets, evaluation metric and implementation details. Then, we compare the performance of the proposed method with the state-of-the-art approaches on different benchmarking datasets.

\section{A. Datasets and evaluation protocols}

We conduct our experiments on several ageing datasets, including MORPH [42], FGNET [43], and AgeDB [44].

MORPH dataset is a large longitudinal collection of 55, 134 mugshot images from 13,617 subjects of different ethnicity in the age range from 16 to 77 years old. More than $96 \%$ of the images in the MORPH dataset are African or European people. Different evaluation protocols are adopted to evaluate the age estimation performance on this dataset. Two popular protocols, namely random splitting (RS) and partial RS, perform a 5fold cross validation on the whole set of images and a subset of 5,493 images from the MORPH dataset, respectively. The subjects of the images used in the partial RS protocol are instances of Caucasian descent to reduce the cross-ethnicity influence. Five-fold subject-exclusive (SE) cross validation protocol is also performed to evaluate the performance of age estimation algorithms. Since there is no identity overlap, this protocol is more challenging. The label distribution of images in the MORPH dataset in terms of gender and ethnicity is significantly unbalanced. Following the works in [45], the MORPH dataset is further randomly partitioned into three nonoverlapped subsets S1, S2 and S3. This splitting makes the Male-Female ratio about three and the White-Black ratio one. Two experiments are performed: 1) training on S1, testing on $\mathrm{S} 2+\mathrm{S} 3$ and 2) training on $\mathrm{S} 2$, testing on $\mathrm{S} 1+\mathrm{S} 3$.

FGNET dataset contains 1,002 images of 82 subjects and the ages range from 0 to 69 years old. The FGNET dataset is challenging due to the large variation in pose, expression and lighting conditions. For the evaluation on the FGNET dataset, we follow the leave-one-person-out (LOPO) protocol [28] in our experiments. In this protocol, all images from one subject are separated for testing and the remained images are used for training. This partitioning is repeated for all 82 subjects in the FGNET dataset and the averaged MAE and CS scores are reported as the final performance.

AgeDB dataset is a in-the-wild collection of 16, 488 images of 568 subjects in the age range form 1 to 101. Following [46], we adopt the RS validation protocol to evaluate the performance of the proposed method.

\section{B. Implementation Details}

Among the wide variety of currently available network architectures, the well-known VGG-16 is the one achieving the best performance on almost all the available datasets for age analysis, as reported in a recent comparative study [22]. We used the pre-trained model (VGG-Face [47]) trained with a large-scale face recognition dataset as the basic backbone of our system. We do not use any other advanced techniques, such as multi-task learning or ensemble learning, in our model. 
Further, we do not use any other external ageing datasets, such as IMDB-WIKI [28], for pre-training. Clearly, by using these techniques and extra training data, better performance can be achieved. We employ an SGD optimiser and set the learning rate, the momentum and the weight decay as 0.001 , 0.9 and 0.0005 , respectively. The learning is decreased by a factor of 10 every 50 epochs. Each model is trained totally in 150 epochs with the batch size of 32 . For the evaluation on the FGNET dataset, due to the limited number of training images, we stop the training when over-fitted. Due to numerical stability, we scale down the ground metric matrix by a certain factor for each dataset. Our algorithm is implemented using the MatConvNe 11 framework on one GTX 1080Ti GPU.

Face alignment and data augmentation are two common preprocessing steps for age estimation. All training and testing images are aligned by the following two steps: Step I - Face detection and facial landmark localisation: the MTCNN face detector [48] is applied to detect the face and extract the corresponding face bounding box and 5 facial landmarks (the left and right centre of the eyes, the nose tip, the left and right corner of the mouth) of each image. Following the work in [28], the detected face bounding box is extended by taking additional $40 \%$ of the width and height of face on all sides. Step II - Face normalisation: In this step, a face image is centred and aligned to a normalised position in order to guarantee all facial landmarks stay at the same position in the image. The facial landmarks are used for the alignment of each face by the method proposed in [49]. Finally, the normalised face is resized to $256 \times 256$ pixels.

In order to relieve the over-fitting problem, we perform standard augmentation techniques during the training stage by random horizontal flipping, rotating $\left( \pm 10^{\circ}\right)$, cropping and adding Gaussian noise to the images. During inference, we use the central cropped image as the input to the network and the expected value over the entries of the output vector $\mathbf{z}$ is considered as the predicted age, i.e. $l_{p}=\sum_{i=1}^{K} i p_{i}$.

We evaluate the performance of an age estimation algorithm in terms of mean absolute error (MAE) and cumulative score (CS) [9]. The MAE is calculated by averaging the absolute error between the predicted age and the ground-truth over the total number of test images. The CS measure is calculated as $\frac{T_{\theta}}{T} \times 100 \%$, where $T_{\theta}$ is the number of test images whose absolute error between the predicted age and the ground-truth is not greater than the threshold $\theta$. In this paper, unless otherwise noted, $\theta$ is set as 5 .

\section{Effect of Hyper-parameters}

There are four hyper-parameters in the proposed $\gamma-$ Wasserstein loss function which should be tuned carefully. To reduce the search space, we first adopted the $p$-Wasserstein loss $(p=1)$ as the ground metric function and performed the partial RS protocol on the MORPH dataset to find the best choices for $\lambda$ and number of iterations in Algorithm 11. We empirically found that $\lambda=1$ and 50 iterations are the best choices. Then, we performed further experiments to determine the hyper-parameters $\rho$ and $\tau$ in the proposed loss function.

\footnotetext{
${ }^{1}$ http://www.vlfeat.org/matconvnet/
}

TABLE I

The Influence of Different PARAmeter Settings on the Proposed LOSS FUNCTION.

\begin{tabular}{|c||c|c|c|c|c|c|c|}
\hline$\tau$ & 1 & 2 & 4 & 6 & 8 & 10 & 12 \\
\hline \hline 0.1 & 2.56 & 2.56 & 2.49 & 2.53 & 2.62 & 2.73 & 2.74 \\
\hline 0.5 & 2.55 & 2.55 & 2.52 & 2.51 & 2.49 & 2.50 & 2.52 \\
\hline 1 & 2.54 & 2.56 & 2.56 & 2.52 & 2.50 & $\mathbf{2 . 4 7}$ & 2.51 \\
\hline 2 & 2.55 & 2.59 & 2.57 & 2.52 & 2.53 & 2.55 & 2.57 \\
\hline
\end{tabular}

Table I] shows MAEs for different combinations of $\rho$ and $\tau$. As seen, the best age estimation performance is achieved using $\rho=10$ and $\tau=1$. As discussed earlier, $\rho$ and $\tau$ should be determined in a such way that the influence of small errors should be amplified. As seen in Fig. 5, for a fixed $\rho$, the effect of small errors is emphasised by choosing a smaller $\tau$. However, as shown in Table I, for optimal performance it is important to control both the "definition" (range) of small errors as well as their influence. This suggests that $\rho$ and $\tau$ need to be optimised jointly.

It should be noted that one can fine-tune the hyper-parameters for each specific dataset. This may achieve marginally better performance. However, this is not practical in real-world applications, where the test images have different characteristics. In this paper, we only evaluate our proposed method with fixed hyper-parameters, fine-tuned on a small set of images, across all the datasets, to verify its effectiveness in practical scenarios.

\section{Comparisons with state-of-the-art algorithms}

We compare the proposed method with a number of stateof-the-art methods in controlled and uncontrolled (in-the-wild) situations. To be fair, we only take deep neural networks-based approaches for comparison. The MAE and CS values are read from the references.

Results in controlled environments: To evaluate the performance of the proposed method, we conduct some experiments on the MORPH dataset which contains facial images captured in a controlled environment. The comparisons under different protocols of the MORPH dataset are summarised in Table II IV] Our method achieves the best MAE performance on the RS and partial RS protocols, which is lower than the best state-ofthe-art method by a margin of $0.33(11.80 \%)$ and $0.18(9.13 \%)$ years, respectively. The results obtained using the SE, S1/S2S3 and S2/S1S3 protocols are more interesting. In contrast to the $\mathrm{RS}$ protocol, there is no identity overlap between training and testing sets in the SE protocol. The training and the test sets are balanced in terms of ethnicity and gender in the S1/S2S3 and S2/S1S3 protocols. The experimental results show that our approach consistently outperforms other methods under these protocols as well. Our method is best among all the approaches, because it gains not only from label distribution learning but also from the proposed similarity-aware ground metric function introduced in the proposed loss function.

Results in uncontrolled environments: The experimental results on the Morph dataset are encouraging. However, images in this dataset are mugshots captured in a controlled environment. 
TABLE II

THE RESUlTS OF THE EVALUATION ON THE MORPH DATASET (RS AND PARTIAL RS PROTOCOLS).

\begin{tabular}{|c|c|c|}
\hline Meth & MAE & $\mathrm{CS}$ \\
\hline \multicolumn{3}{|c|}{ Partial RS Protocol } \\
\hline AGEn 45 & 4.34 & - \\
\hline $\operatorname{DEX}|28|$ & 3.25 & - \\
\hline DNCL [27| & 2.85 & 83.8 \\
\hline $\mathrm{DRF}\lceil 50\rceil$ & 2.80 & 85.6 \\
\hline $\mathrm{DC}[32]$ & 2.57 & 89.6 \\
\hline TV Distance & 2.89 & $\overline{86} . \overline{1}$ \\
\hline Cross Entropy (CE) & 2.65 & 88.3 \\
\hline KL Divergence & 2.56 & 89.1 \\
\hline$p$-Wasserstein & 2.50 & 89.3 \\
\hline$\gamma-\overline{\text { Wasserstein }}$ & $2.4 \overline{7}$ & $\overline{90.4}$ \\
\hline \multicolumn{3}{|c|}{ RS Protocol } \\
\hline $\mathrm{C} 3 \mathrm{AE}[51]$ & 2.78 & - \\
\hline DLDL $2 \mid$ & 2.42 & - \\
\hline CE-MV [52] & 2.41 & 90.1 \\
\hline DRF $|50|$ & 2.14 & 91.3 \\
\hline DLDL-v2 31 & 1.97 & - \\
\hline $\mathrm{DC}[32]$ & 1.88 & 94.3 \\
\hline TV Distance & $2.1 \overline{4}$ & $\overline{90 . \overline{4}}$ \\
\hline Cross Entropy (CE) & 1.96 & 92.7 \\
\hline KL Divergence & 1.90 & 93.1 \\
\hline$p$-Wasserstein & 1.89 & 93.1 \\
\hline$\gamma-\overline{\text { Wasserstein }}$ & 1.79 & $\overline{94 . \overline{4}}$ \\
\hline
\end{tabular}

We are more interested in assessing the performance of age estimation systems on images taken in uncontrolled environments which are more challenging. So, we conducted further experiments on two uncontrolled datasets, i.e. FGNET and AgeDB datasets. Compared with the FGNET dataset, the images in the AgeDB dataset are captured under more sever shooting situations, where more different poses, noise and occlusions, etc. occur.

The results on the FGNET and AgeDB datasets are given in Table $\mathrm{V}$ and $\mathrm{VI}$ respectively. As the AgeDB dataset is relatively new, there are a very few evaluations of age estimation methods reported in the literature. Compared to the results on the MORPH dataset, the accuracy of all age estimation algorithms degraded. This degradation in the performance can be attributed to the uncontrolled external factors which include makeup and expressions in the face, variable lighting conditions, changes in head pose, resolution of the facial images etc. Our proposed method still achieves the lowest MAE and highest CS score among the existing approaches on both FGNET and AgeDB datasets. This suggests that our method has a better generalisation ability to unseen scenarios compared with other existing methods.

Some examples of face images in the wild (from AgeDB) with good and poor age estimation accuracy by the proposed $\gamma$-Wasserstein are shown in Fig. 7 . As seen, the low accurate predictions are mostly caused by the difficult conditions created by very bad illumination, different kinds of occlusion, blurring, makeup etc.
TABLE III

THE RESUlTS OF THE EVALUATION ON THE MORPH DATASET (SE PROTOCOL).

\begin{tabular}{|c|c|c|}
\hline Method & MAE & CS (\%) \\
\hline Demographic features $|53|$ & 3.60 & - \\
\hline Multi-Task |54| & 3.00 & 85.3 \\
\hline Soft-Ranking |55| & 2.83 & - \\
\hline CE-MV [52] & 2.80 & - \\
\hline Xie et al. & 2.69 & - \\
\hline TV Distance & 3.12 & $\overline{84} . \overline{0}$ \\
\hline Cross Entropy (CE) & 2.94 & 86.5 \\
\hline KL Divergence & 2.82 & 87.2 \\
\hline$p$-Wasserstein & 2.78 & 87.8 \\
\hline$\gamma-\overline{\text { Wasserstein }}$ & $2 . \overline{71}$ & $\overline{88.1}$ \\
\hline
\end{tabular}

TABLE IV

THE RESUlTS OF EVALUATION ON THE MORPH DATASET (S1/S2+S3 AND S2/S1+S3 PROTOCOLS).

\begin{tabular}{|c|c|c|c|c|c|c|}
\hline & \multicolumn{2}{|c|}{ S1/S2+S3 } & \multicolumn{2}{|c|}{$\mathbf{S} 2 / \mathbf{S} 1+\mathbf{S 3}$} & \multicolumn{2}{|c|}{ Average } \\
\hline Method & MAE & CS $(\%)$ & MAE & CS (\%) & MAE & CS $(\%)$ \\
\hline Soft-Softmax $|56|$ & 3.24 & - & 3.03 & - & 3.14 & - \\
\hline D2C $[57]$ & 3.06 & - & 3.05 & - & 3.06 & - \\
\hline Wan et al. |58| & 3.08 & - & 2.78 & - & 2.93 & - \\
\hline AGEn $|45|$ & 3.04 & - & 2.68 & - & 2.86 & 84.0 \\
\hline Xie et al. $|46|$ & 2.80 & - & 2.81 & - & 2.81 & - \\
\hline $\mathrm{DC}|32| \ldots$ & 2.80 & 87.2 & 2.8 & 85.2 & 2.8 & 86.2 \\
\hline TV Distance & $\overline{2} . \overline{96}$ & $\overline{85.2}$ & 3.01 & $\overline{8} 4.9$ & $2 . \overline{98}$ & $\overline{85} . \overline{0}$ \\
\hline Cross Entropy (CE) & 2.88 & 86.5 & 2.87 & 86.6 & 2.87 & 86.5 \\
\hline KL Divergence & 2.78 & 87.3 & 2.79 & 87.3 & 2.79 & 87.3 \\
\hline$p$-Wasserstein & 2.75 & 87.4 & 2.75 & 87.5 & 2.75 & 87.5 \\
\hline$\overline{\gamma-\bar{W}}-\overline{\text { asserstein }}$ & $\overline{2.69}$ & $8 \overline{8} .2$ & 2.70 & $\overline{88.2}$ & $2 . \overline{70}$ & $\overline{88 .} \overline{2}$ \\
\hline
\end{tabular}

TABLE V

Evaluation on the FGNET Dataset (LOPO Protocol).

\begin{tabular}{|c|c|c|}
\hline Method & MAE & CS (\%) \\
\hline DEX |28] & 4.63 & - \\
\hline AGEn $|45|$ & 4.34 & 76.0 \\
\hline Ranking-CNN [59] & 4.13 & - \\
\hline CE-MV [52] & 4.10 & 78.5 \\
\hline C3AE [51] & 4.09 & - \\
\hline DNCL 27 & 3.71 & 81.8 \\
\hline Xie et al. & 3.58 & 78.3 \\
\hline DRF $|50|$ & 3.47 & 87.3 \\
\hline TV Distance & 3.65 & $\overline{79} . \overline{8}$ \\
\hline Cross Entropy (CE) & 3.52 & 81.3 \\
\hline KL Divergence & 3.48 & 82.0 \\
\hline$p$-Wasserstein & 3.52 & 81.5 \\
\hline$\gamma-\overline{\text { Wasserstein }}$ & 3.41 & $82 . \overline{9}$ \\
\hline
\end{tabular}

\section{E. Evaluation of different loss functions}

To validate the effectiveness of the proposed loss function, we compare it with several existing loss functions: the TV distance, the KL divergence, $p$-Wasserstein metric and the proposed $\gamma$ Wasserstein loss function. We further, compare with multi-class 
TABLE VI

Evaluation on the AgeDB DATASET (RS Protocol). The MAE AND CS VAlues ARE READ FROM [46].

\begin{tabular}{|c|c|c|}
\hline Method & MAE & CS $(\%)$ \\
\hline AGEn [45] & 6.22 & 53.5 \\
\hline CE-MV [52] & 5.87 & 57.2 \\
\hline Xie et al. & 5.58 & 53.2 \\
\hline TV Distance & $5 . \overline{7} 5$ & $57 . \overline{8}$ \\
\hline Cross Entropy (CE) & 5.41 & 59.2 \\
\hline KL Divergence & 5.29 & 61.4 \\
\hline$p$-Wasserstein & 5.20 & 61.8 \\
\hline$\gamma-\overline{\text { Wasserstein }}$ & 5.16 & $\overline{62.9}$ \\
\hline
\end{tabular}

TABLE VII

The INFLUENCE OF VALUes of PARAMETER $p$ IN THE $p$-WASSERSTEIN LOSS FUNCTION.

\begin{tabular}{|c||c|c|c|c|c|c|c|c|}
\hline$p$ & 0.7 & 0.8 & 0.9 & 1 & 1.1 & 1.2 & 1.3 & 2 \\
\hline \hline MAE & 4.01 & 2.78 & 2.50 & 2.50 & 2.49 & 2.52 & 8.12 & NaN \\
\hline
\end{tabular}

classification approach where the cross entropy (CE) is used as the loss function. First, we performed the partial RS protocol on the MORPH dataset to find the best value of hyper-parameter $p$ in the $p$-Wasserstein loss function. We empirically found the training algorithm explodes for $p \geq 2$. This is due to the appearance of large values assigned to far points by the ground metric function. Table $\overline{\mathrm{VII}}$ shows MAEs for different values of $p$. As seen, the best age estimation performance is achieved using values of $p$ close to 1 .

The experimental results are shown in bottom part of Tables II-VI. TV distance has the highest MAE among existing loss functions. Comparing the results of KL divergence with those of $\mathrm{T}$ distance, the increase of age estimation accuracy is apparent. The accuracy further increases by utilising the $p$-Wasserstein algorithm, but the reduction is relatively small with respect to the KL divergence. Finally, we observe that the best values of MAE and of the CS score are achieved when the $\gamma$-Wasserstein loss is used for training the model.

\section{F. Computational complexity}

The proposed and competing methods have the same computational complexity at the inference stage. However, at the training stage, the computational complexity of our proposed method is higher than the divergence-based methods. For solving the minimisation problem (1), the gradient of the loss function $\ell$ should be computed. Using a divergence bases loss function, e.g. KL divergence or TV distance, the gradient is directly obtained via Eq. (4) or Eq. (5). This is with the computational complexity of order $\mathcal{O}(n)$. However, for computing the gradient using an optimal transport loss function, e.g. $p$-Wasserstein or $\gamma$-Wasserstein loss function, we first need to solve the minimisation (10) using the SinkhornKnopp algorithm. This has computational complexity of the order of $\mathcal{O}\left(n^{2}\right)|60|$. Then, the gradient is obtained via Eq. (11) with computational complexity $\mathcal{O}\left(n^{2} \log (n)\right)$.

\section{G. Discussion}

1) Effect of Age Group: To evaluate the accuracy of the proposed method at each age class, the age of all images within each class is estimated by our proposed algorithm. This experiment is conducted on the validation set of the AgeDB and MORPH datasets by following the RS protocol. Fig. 8 illustrates the performance of our age estimation algorithm for different age classes in each dataset. The solid line indicates the average predicted age in each age class and the red bars represent the standard deviation of the predicted age at each class. The dashed line is a reference to show where accurate predictions must lie.

It can be observed that the average accuracy of our proposed method differs at different ages. Generally, the gap between the estimated ages and ground-truth ones increases towards older and younger ages. The system tends to have accurate performance in middle ages. The age of younger subjects is slightly over-estimated. This is more pronounced for the subjects in the AgeDB dataset. Starting from approximately the age of 60 years old, the very accurate performance of our system elsewhere begins to diverge from the correct prediction line. This observation may be due to the limited number of training images in the older age categories. Moreover, a texture change is the most perceptible change during adulthood. Texture changes appearing at very old ages are very fine and difficult to recognise. For this reason, the precise age estimation for older people is more difficult.

Fig. 9 shows the distribution of ages predicted by our proposed algorithm for different evaluation datasets. The solid and dashed lines show the age distributions of predicted ages and ground-truth, respectively. As can be seen, the model trained by our proposed algorithm follows the age distribution of the the MORPH and AgeDB datasets. Our system closely models the distribution of the MORPH dataset, which is bimodal. However, it is not able to fit the ground-truth distribution of the AgeDB dataset. This could be due to in-the-wild faces in the AgeDB dataset which lead to degradation in the performance.

2) Error Distribution: As the MAE and CS measures only reflect the age prediction errors over all test data, it is important to know how certain the model is in the age prediction and how far off it might be. Fig. 10 shows the distribution of the prediction errors (between estimated ages and target ages) of two models trained by $\gamma$-Wasserstein and $p$-Wasserstein on the MORPH dataset under the RS protocol. As can be seen, the error distribution for both models approaches the Gaussian distribution and the maximum error between the estimated and the target ages is around 10 years. As showed in Fig. 7, this error might be due to illumination conditions, face occlusion, blurring, makeup etc Moreover, Fig. 10 illustrates how using a different ground metric function for the optimum transport based loss function will result in different patterns for the error distribution curves. As can be observed, the standard deviation of the error distribution of the model trained by the $p$-Wasserstein loss function is higher. Furthermore, the mean of the distribution is slightly shifted to the right.

3) Cross-dataset evaluation: There are many demographic factors, such as gene, gender, ethnicity etc., and many other 

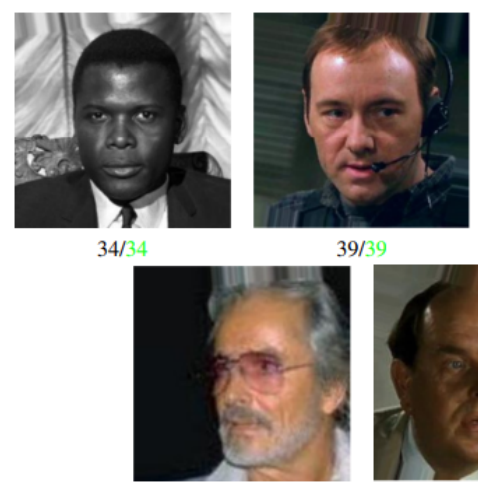

$56 / 62$
$39 / 39$

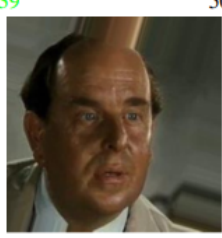

$43 / 52$

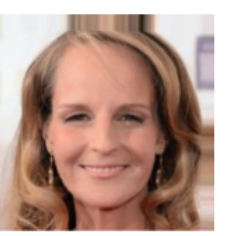

$50 / 51$

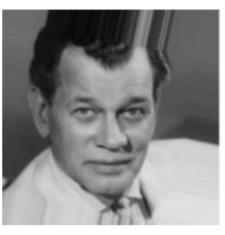

$55 / 56$

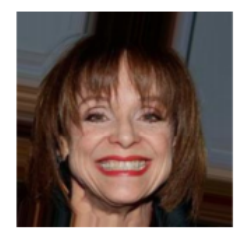

$70 / 72$

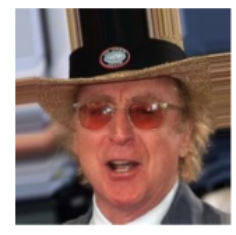

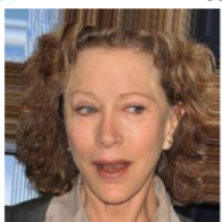

$65 / 56$

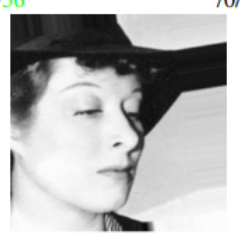

28/40

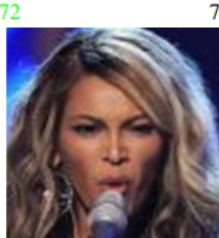

$69 / 41$

Fig. 7. Ground-truth/Estimated age of exemplar images from the AgeDB dataset with good and bad age estimation achieved by the proposed method.

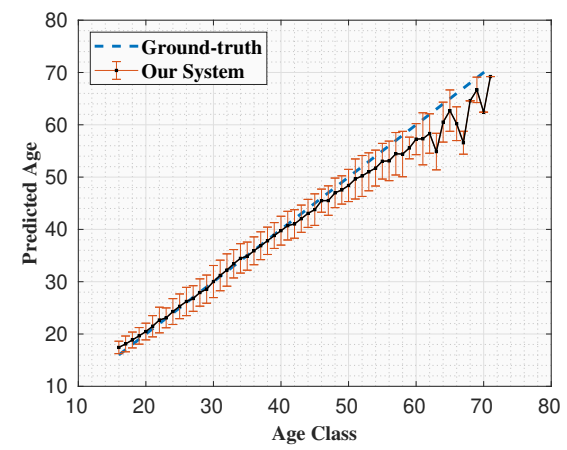

(a)

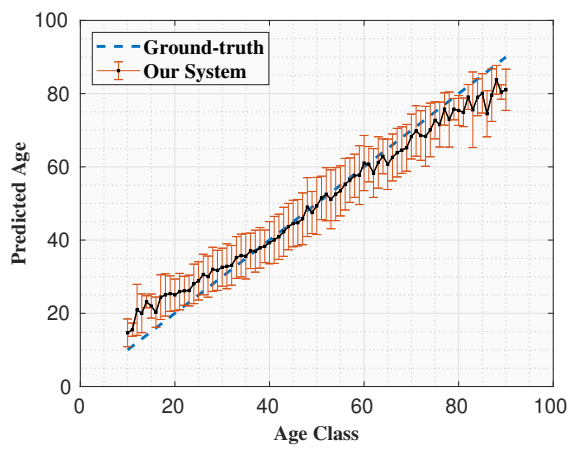

(b)

Fig. 8. Average predicted age at each age class for different datasets. (a) MORPH. (b) AgeDB.

external factors, such as illumination conditions, image quality, makeup, lifestyle, cosmetic surgery, etc., which may confound the training process, if the distribution of the training images is skewed with respect to these factors [61]. For instance, the distribution of images in the MORPH dataset in terms of ethnicity is significantly unbalanced. More than $96 \%$ of the images in the MORPH dataset are African or European people. In the AgeDB dataset, a bias may be further introduced to the dataset by makeup covering age signs such as wrinkles and age spots. Since a neural network efficiently learns a data distribution, the performance of the trained models is prone to be biased to the distribution of the training data. These biases associated with the ageing datasets cause the trained models

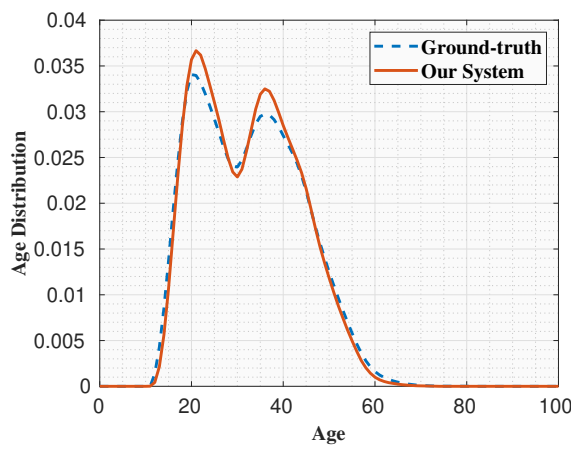

(a)

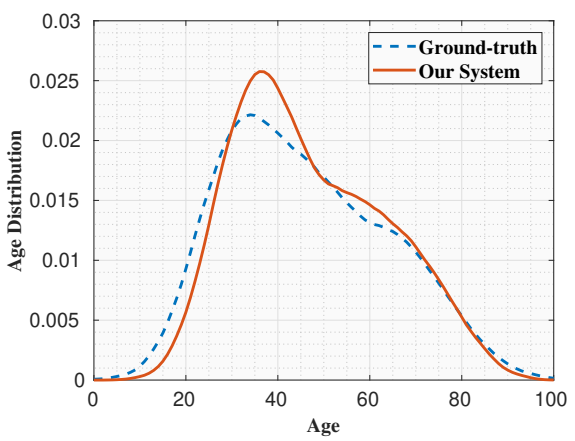

(b)

Fig. 9. Distribution of the predicted ages for different datasets. (a) MORPH. (b) AgeDB.

to perform best with test data which is similar to that used to train the model, and to generalise poorly to unseen data, if the test data has a different distribution.

In this section, we reveal the existence of a dataset bias in the ageing datasets via cross-dataset evaluation, where the test and training sets have different data distributions and characteristics. Specifically, we evaluate the performance of the trained models on a dataset other than it was trained for (trained on the AgeDB dataset and tested on the MORPH and FGNET datasets, and trained on the MORPH dataset and tested on the AgeDB and FGNET datasets).

The results for the intra-dataset and cross-dataset evaluations are given in Table VIII The numbers in the bold font represent 


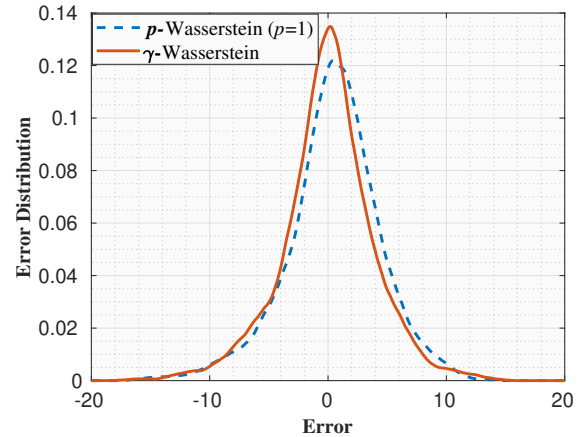

Fig. 10. Distribution of the errors on the MORPH dataset (RS protocol). The red line has a standard deviation of 3.8632 and a mean of -0.02 . The blue line has a standard deviation of 3.9067 and a mean of 0.47 .

TABLE VIII

INTRA AND CROSS-DATASET EVALUATION. INTRA-DATASET RESULTS ARE IN BOLD.

\begin{tabular}{|l|cc|cc|cc|}
\hline & \multicolumn{4}{|c|}{ Test Datasets } \\
\hline \hline & \multicolumn{2}{|c|}{ FGNET } & \multicolumn{2}{c|}{ MORPH } & \multicolumn{2}{c|}{ AgeDB } \\
\hline \hline Training Dataset & \multicolumn{2}{|c|}{ MAE } & CS (\%) & MAE & CS (\%) & MAE CS (\%) \\
\hline MORPH & 7.01 & 46.15 & $\mathbf{1 . 7 9}$ & $\mathbf{9 4 . 4}$ & 10.65 & 33.19 \\
AgeDB & 9.82 & 28.94 & 5.72 & 55.60 & $\mathbf{5 . 1 6}$ & $\mathbf{6 2 . 9 1}$ \\
\hline
\end{tabular}

the intra-dataset results. Compared with intra-dataset evaluation, the accuracy of our age estimation algorithm in the cross-dataset evaluation is significantly degraded. In Fig. 11, we show the distribution of the predicted ages for the two models trained on MORPH and LAP datasets, respectively. Compared with the distribution of the predicted ages under the intra-domain evaluation (see Fig. 9), the trained models are not able to follow the ground-truth distributions in the cross-dataset evaluation. These results confirm that the age estimation performance is severely affected by the dataset bias. This calls for designing alternative leaning schemes which make the trained model agnostic to the dataset bias and provide models which have the ability of overcoming the bias between the training and testing sets. The future work in this research area will need to develop the key elements of age estimation system training, such as a loss function, learning rate policy and architecture components, so that the trained model can render an age estimation solution which is more domain invariant than existing methods.

4) Important face regions: Motivated by [28], we conduct the following experiment in order to determine the most important parts of the face image to the overall age estimation accuracy. In each test image, a block within the face area is replaced by the mean image. The block size used is $20 \times 20$ pixels. The MAE on the validation images of the MORPH dataset for each of the block occlusions is given in Fig. 12 As seen, the occlusion of different parts of the face frame has a different impact on the age estimation performance. The area from the eyes to the chin and that between two ears through the centre of the face are the most informative parts of the face for the age estimation. Occluding the area between the bottom of the nose and the upper lip increases the MAE the most. The next important parts are the regions close

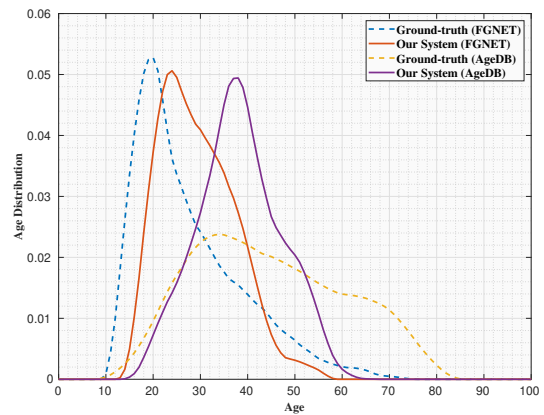

(a)

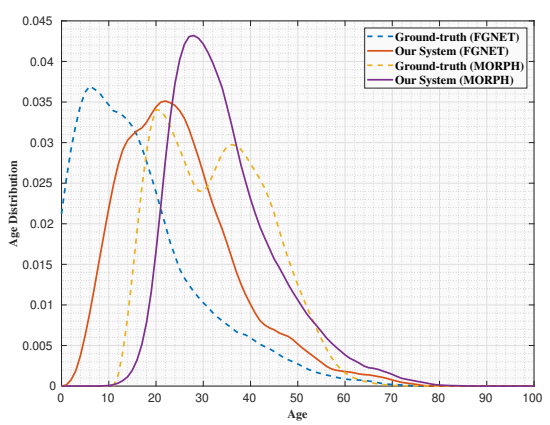

(b)

Fig. 11. Distribution of the predicted ages for two models: (a) Trained on the MORPH dataset, (b) Trained on the AgeDB dataset.
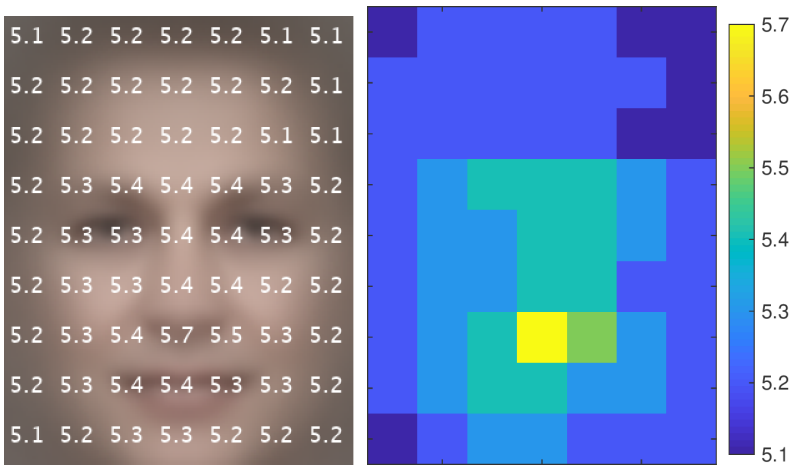

Fig. 12. Impact of the block occlusion of face region with size of $20 \times 20$ pixels on the age estimation accuracy (MAE).

to eyes and lips. Surprisingly, the area above the eyebrows passing through forehead does not have much impact on the performance, although this area has apparent ageing patterns. In fact, due to in-the-wild nature of the training set, the top part of a large proportion of training images are occluded by hair, hat etc. Further, cosmetic surgery also hides these ageing signs. Therefore, the trained models may not be sensitive to this area of the face for estimating the age.

\section{CONCLUSION}

In this paper, we proposed an optimal transport-based loss function for the age estimation within a label distribution learning framework. Adopting the notion of optimal transport to deal with the label distribution task has two advantages. Firstly, the optimal transport has been shown to have the potential 
to measure the distance between two label distributions properly. This, in particular, avoids the common problem with information divergence-based measures, such as KL divergence and TV distance, to gauge the distance between two label distributions, which do not overlap. Secondly, by devising the ground metric function for the optimal transport so that it closely reflects the semantic similarity of neighbouring classes, the learnt age estimation model becomes more effective. Extensive evaluations on the FGNET, MORPH, AgeDB datasets confirm the merits of the proposed approach in terms of delivering better accuracy of age predictions. While this work primarily focuses on a label distribution learning framework to establish its virtue for the age estimation problem, it can be applied to other machine learning tasks where there is some kind of similarity among classes. We are currently investing this in relation to other applications. Furthermore, we note that our original formulation has a limitation which makes it impractical for comparing distributions supported on different topological spaces. Applying our model to such learning tasks is an important future direction of this work.

\section{ACKNOWLEDGMENT}

This work was supported in part by the EPSRC Programme Grant (FACER2VM) EP/N007743/1 and the EPSRC/dstl/MURI project EP/R018456/1.

\section{REFERENCES}

[1] X. Geng, "Label distribution learning," IEEE Transactions on Knowledge and Data Engineering, vol. 28, no. 7, pp. 1734-1748, July 2016.

[2] B. Gao, C. Xing, C. Xie, J. Wu, and X. Geng, "Deep label distribution learning with label ambiguity," IEEE Transactions on Image Processing, vol. 26, no. 6, pp. 2825-2838, June 2017.

[3] I. Sason and S. Verdú, " $f$-divergence inequalities," IEEE Transactions on Information Theory, vol. 62, no. 11, pp. 5973-6006, 2016.

[4] C. Villani, Optimal Transport, Old and New. Springer-Verlag Berlin Heidelberg, 7 2209, vol. 338.

[5] M. Cuturi, "Sinkhorn distances: Light-speed computation of optimal transport," in Advances in Neural Information Processing Systems (NIPS), 2013, pp. 2292-2300.

[6] S. Kolouri, S. R. Park, M. Thorpe, D. Slepcev, and G. K. Rohde, "Optimal mass transport: Signal processing and machine-learning applications," IEEE Signal Processing Magazine, vol. 34, no. 4, pp. 43-59, July 2017.

[7] C. Frogner, C. Zhang, H. Mobahi, M. Araya-Polo, and T. Poggio, "Learning with a wasserstein loss," in Advances in Neural Information Processing Systems (NIPS), 2015, pp. 2053-2061.

[8] A. Genevay, G. Peyre, and M. Cuturi, "Learning generative models with sinkhorn divergences," in Proceedings of Conference on Artificial Intelligence and Statistics, ser. Proceedings of Machine Learning Research, vol. 84, Playa Blanca, Lanzarote, Canary Islands, 09-11 Apr 2018, pp. 1608-1617.

[9] G. Guo, , Y. Fu, and T. S. Huang, "Human age estimation using bioinspired features," in IEEE/CVF Conference on Computer Vision and Pattern Recognition (CVPR), June 2009, pp. 112-119.

[10] A. Lanitis, C. Draganova, and C. Christodoulou, "Comparing different classifiers for automatic age estimation," IEEE Transactions on Systems, Man, and Cybernetics, Part B (Cybernetics), vol. 34, no. 1, pp. 621-628, Feb 2004.

[11] S. Wang, D. Tao, and J. Yang, "Relative attribute svm+ learning for age estimation," IEEE Transactions on Cybernetics, vol. 46, no. 3, pp. 827-839, 2016.

[12] A. Akbari, M. Trocan, and B. Granado, "Sparse recovery-based error concealment," IEEE Transactions on Multimedia, vol. 19, no. 6, pp $1339-1350,2017$.

[13] A. Akbari, M. Trocan, S. Sanei, and B. Granado, "Joint sparse learning with nonlocal and local image priors for image error concealment," IEEE Transactions on Circuits and Systems for Video Technology, vol. 30, no. 8, pp. 2559-2574, 2020.
[14] A. Akbari, M. Trocan, and B. Granado, "Joint-domain dictionary learningbased error concealment using common space mapping," in International Conference on Digital Signal Processing (DSP), 2017, pp. 1-5.

[15] — "Image error concealment using sparse representations over a trained dictionary," in Picture Coding Symposium (PCS), 2016, pp. 1-5.

[16] H. Liao, "Facial age feature extraction based on deep sparse representation," Multimedia Tools and Applications, vol. 78, pp. 2181-2197, 2019.

[17] x. shu, J. Tang, Z. Li, H. Lai, L. Zhang, and S. Yan, "Personalized age progression with bi-level aging dictionary learning," IEEE Transactions on Pattern Analysis and Machine Intelligence, vol. 40, no. 4, pp. 905-917, 2018.

[18] S. S. Khalid, M. Awais, Z. H. Feng, C. H. Chan, A. Farooq, A. Akbari, and J. Kittler, "Resolution invariant face recognition using a distillation approach," IEEE Transactions on Biometrics, Behavior, and Identity Science, vol. 2, no. 4, pp. 410-420, 2020.

[19] S. Fatemifar, M. Awais, A. Akbari, and J. Kittler, "A stacking ensemble for anomaly based client-specific face spoofing detection," in 2020 IEEE International Conference on Image Processing (ICIP), 2020, pp. 13711375.

[20] M. Bashar, A. Akbari, K. Cumanan, H. Q. Ngo, A. G. Burr, P. Xiao, and M. Debbah, "Deep learning-aided finite-capacity fronthaul cell-free massive mimo with zero forcing," in IEEE International Conference on Communications (ICC), 2020, pp. 1-6.

[21] M. Bashar, A. Akbari, K. Cumanan, H. Q. Ngo, A. G. Burr, P. Xiao, M. Debbah, and J. Kittler, "Exploiting deep learning in limited-fronthaul cell-free massive mimo uplink," IEEE Journal on Selected Areas in Communications (JSAC), vol. 38, no. 8, pp. 1678-1697, 2020.

[22] V. Carletti, A. Greco, G. Percannella, and M. Vento, "Age from faces in the deep learning revolution," IEEE Transactions on Pattern Analysis and Machine Intelligence, pp. 1-1, Apr 2019.

[23] X. Wang, R. Guo, and C. Kambhamettu, "Deeply-learned feature for age estimation," in IEEE Winter Conference on Applications of Computer Vision, Jan 2015, pp. 534-541.

[24] C. Li, Q. Liu, W. Dong, X. Zhu, J. Liu, and H. Lu, "Human age estimation based on locality and ordinal information," IEEE Transactions on Cybernetics, vol. 45, no. 11, pp. 2522-2534, 2015.

[25] Z. Niu, M. Zhou, L. Wang, X. Gao, and G. Hua, "Ordinal regression with multiple output cnn for age estimation," in IEEE/CVF Conference on Computer Vision and Pattern Recognition (CVPR), June 2016, pp. 4920-4928.

[26] S. Chen, C. Zhang, M. Dong, J. Le, and M. Rao, "Using ranking-cnn for age estimation," in IEEE/CVF Conference on Computer Vision and Pattern Recognition (CVPR), July 2017, pp. 742-751.

[27] L. Zhang, Z. Shi, M. Cheng, Y. Liu, J. Bian, J. T. Zhou, G. Zheng, and Z. Zeng, "Nonlinear regression via deep negative correlation learning," IEEE Transactions on Pattern Analysis and Machine Intelligence, pp. $1-1,2019$.

[28] R. Rothe, R. Timofte, and L. Van Gool, "Deep expectation of real and apparent age from a single image without facial landmarks," International Journal of Computer Vision, vol. 126, no. 2, pp. 144-157, Apr 2018.

[29] K. Zhang, C. Gao, L. Guo, M. Sun, X. Yuan, T. X. Han, Z. Zhao, and B. Li, "Age group and gender estimation in the wild with deep ror architecture," IEEE Access, vol. 5, pp. 22 492-22 503, 2017.

[30] Z. He, X. Li, Z. Zhang, F. Wu, X. Geng, Y. Zhang, M. Yang, and Y. Zhuang, "Data-dependent label distribution learning for age estimation," IEEE Transactions on Image Processing, vol. 26, no. 8, pp. 3846-3858, Aug 2017.

[31] B.-B. Gao, H.-Y. Zhou, J. Wu, and X. Geng, "Age estimation using expectation of label distribution learning," in Proceedings of International Joint Conference on Artificial Intelligence (IJCAI), 7 2018, pp. 712-718.

[32] A. Akbari, M. Awais, Z. Feng, A. Farooq, and J. Kittler, "Distribution cognisant loss for cross-database facial age estimation with sensitivity analysis," IEEE Transactions on Pattern Analysis and Machine Intelligence, pp. 1-1, 2020.

[33] — "A flatter loss for bias mitigation in cross-dataset facial age estimation," in International Conference on Pattern Recognition (ICPR), 2021.

[34] J. Qian, W. K. Wong, H. Zhang, J. Xie, and J. Yang, "Joint optimal transport with convex regularization for robust image classification," IEEE Transactions on Cybernetics, pp. 1-12, 2020.

[35] N. Courty, R. Flamary, A. Habrard, and A. Rakotomamonjy, "Joint distribution optimal transportation for domain adaptation," in Advances in Neural Information Processing Systems (NIPS), 2017, pp. 3730-3739.

[36] J. Liu, J. He, Y. Xie, W. Gui, Z. Tang, T. Ma, J. He, and J. P. Niyoyita, "Illumination-invariant flotation froth color measuring via wasserstein 
distance-based cyclegan with structure-preserving constraint," IEEE Transactions on Cybernetics, pp. 1-14, 2020.

[37] X. Geng, C. Yin, and Z. Zhou, "Facial age estimation by learning from label distributions," IEEE Transactions on Pattern Analysis and Machine Intelligence, vol. 35, no. 10, pp. 2401-2412, Oct 2013.

[38] O. Pele and M. Werman, "Fast and robust earth mover's distances," in International Conference on Computer Vision (ICCV), 2009, pp. 460-467.

[39] R. Sinkhorn and P. Knopp, "Concerning nonnegative matrices and doubly stochastic matrices." Pacific Journal of Mathematics, vol. 21, no. 2, pp 343-348, 1967.

[40] Z.-H. Feng, J. Kittler, M. Awais, P. Huber, and X.-J. Wu, "Wing loss for robust facial landmark localisation with convolutional neural networks," in IEEE/CVF Conference on Computer Vision and Pattern Recognition (CVPR), June 2018.

[41] X. Wang, L. Bo, and L. Fuxin, "Adaptive wing loss for robust face alignment via heatmap regression," in IEEE/CVF Conference on Computer Vision (ICCV), 2019, pp. 6970-6980.

[42] K. Ricanek and T. Tesafaye, "Morph: a longitudinal image database of normal adult age-progression," in International Conference on Automatic Face and Gesture Recognition (FGR), Apr 2006, pp. 341-345.

[43] G. Panis, A. Lanitis, N. Tsapatsoulis, and T. F. Cootes, "Overview of research on facial ageing using the fg-net ageing database," IET Biometrics, vol. 5, no. 2, pp. 37-46, May 2016.

[44] S. Moschoglou, A. Papaioannou, C. Sagonas, J. Deng, I. Kotsia, and S. Zafeiriou, "Agedb: The first manually collected, in-the-wild age database," in IEEE Conference on Computer Vision and Pattern Recognition Workshops (CVPRW), July 2017, pp. 1997-2005.

[45] Z. Tan, J. Wan, Z. Lei, R. Zhi, G. Guo, and S. Z. Li, "Efficient group-n encoding and decoding for facial age estimation," IEEE Transactions on Pattern Analysis and Machine Intelligence, vol. 40, no. 11, pp. 2610-2623, Nov 2018.

[46] J. Xie and C. Pun, "Chronological age estimation under the guidance of age-related facial attributes," IEEE Transactions on Information Forensics and Security, vol. 14, no. 9, pp. 2500-2511, Sep 2019.

[47] O. M. Parkhi, A. Vedaldi, and A. Zisserman, "Deep face recognition," in British Machine Vision Conference, 2015.

[48] K. Zhang, Z. Zhang, Z. Li, and Y. Qiao, "Joint face detection and alignment using multitask cascaded convolutional networks," IEEE Signal Processing Letters, vol. 23, no. 10, pp. 1499-1503, Oct 2016.

[49] Y. Wen, K. Zhang, Z. Li, and Y. Qiao, "A discriminative feature learning approach for deep face recognition," in European Conference on Computer Vision (ECCV). Springer International Publishing, 2016 , pp. 499-515.

[50] W. Shen, Y. Guo, Y. Wang, K. Zhao, B. Wang, and A. L. Yuille, "Deep differentiable random forests for age estimation," IEEE Transactions on Pattern Analysis and Machine Intelligence, pp. 1-1, Aug 2019.

[51] C. Zhang, S. Liu, X. Xu, and C. Zhu, "C3AE: exploring the limits of compact model for age estimation," in IEEE/CVF Conference on Computer Vision and Pattern Recognition (CVPR), June 2019.

[52] H. Pan, H. Han, S. Shan, and X. Chen, "Mean-variance loss for deep age estimation from a face," in IEEE Conference on Computer Vision and Pattern Recognition, June 2018, pp. 5285-5294.

[53] H. Han, C. Otto, X. Liu, and A. K. Jain, "Demographic estimation from face images: Human vs. machine performance," IEEE Transactions on Pattern Analysis and Machine Intelligence, vol. 37, no. 6, pp. 1148-1161, June 2015.

[54] H. Han, A. K. Jain, F. Wang, S. Shan, and X. Chen, "Heterogeneous face attribute estimation: A deep multi-task learning approach," IEEE Transactions on Pattern Analysis and Machine Intelligence, vol. 40, no. 11, pp. 2597-2609, Nov 2018.

[55] X. Zeng, C. Ding, Y. Wen, and D. Tao, "Soft-ranking label encoding for robust facial age estimation," CoRR, vol. abs/1906.03625, 2019.

[56] X. Geng, Q. Wang, and Y. Xia, "Facial age estimation by adaptive label distribution learning," in International Conference on Pattern Recognition (ICPR), Aug 2014, pp. 4465-4470.

[57] K. Li, J. Xing, W. Hu, and S. J. Maybank, "D2C: Deep cumulatively and comparatively learning for human age estimation," Pattern Recognition, vol. 66, pp. 95-105, June 2017.

[58] J. Wan, Z. Tan, Z. Lei, G. Guo, and S. Z. Li, "Auxiliary demographic information assisted age estimation with cascaded structure," IEEE Transactions on Cybernetics, vol. 48, no. 9, pp. 2531-2541, Sep 2018.

[59] S. Chen, C. Zhang, and M. Dong, "Deep age estimation: From classification to ranking," IEEE Transactions on Multimedia, vol. 20, no. 8, pp. 2209-2222, Aug 2018.

[60] P. Dvurechensky, A. Gasnikov, and A. Kroshnin, "Computational optimal transport: Complexity by accelerated gradient descent is better than by sinkhorn's algorithm," in Proceedings of International Conference on Machine Learning (ICML), 2018, pp. 1366-1375.

[61] A. Akbari, M. Awais, and J. Kittler, "Sensitivity of age estimation systems to demographic factors and image quality: Achievements and challenges," in IEEE International Joint Conference on Biometrics (IJCB), 2020, pp. $1-6$.

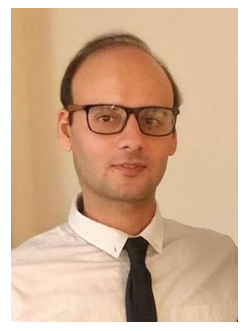

Ali Akbari (SM'15, M'18) received the M.S. degree in Electrical Engineering from the Shiraz University of Technology, Iran in 2012 and the $\mathrm{PhD}$ degree in Telecommunications at the Institut supérieur d'électronique de Paris (ISEP), Sorbonne Université, Paris, France in March 2018. Since July 2018, he joined the Centre for Vision, Speech and Signal Processing (CVSSP), University of Surrey, UK as a research fellow to enrich his experiences in the field of face recognition. He also served as a member of the Editorial Board of the IEEE Open Journal of Circuits and Systems (OJCAS) since 2019. His research interests include image and video processing, computer vision, deep learning and dictionary learning

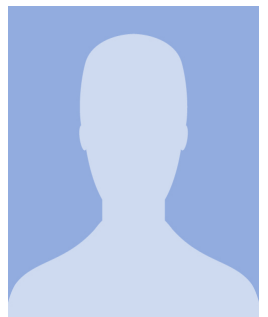

Muhammad Awais received the B.Sc. degree in mathematics and physics from the AJK University in 2001, B.Sc. degree in computer engineering from UET Taxila in 2005, M.Sc in signal processing and machine intelligence and $\mathrm{PhD}$ in machine learning from the University of Surrey in 2008 and 2011. He is currently a senior research fellow at the Centre for Vision, Speech and Signal Processing (CVSSP) at the University of Surrey. His research interests include image processing, computer vision, pattern recognition, machine learning and deep learning.

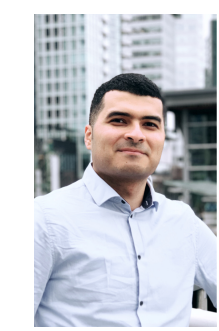

Soroush Fatemifar received the B.Eng. degree in Computer Hardware Engineering from the Shiraz University, Shiraz, Iran., in 2015, the M.Sc. degree in Information Technology from the University of Tehran, Tehran, Iran, in 2017.He is a current Ph.D student in Electrical Engineering at the University of Surrey, Guildford, U.K. His research interests cover the deep learning, machine learning, biometrics, anomaly detection, and also ensemble learning.

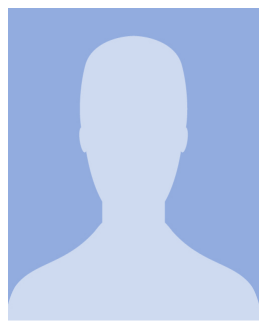

Syed Safwan Khalid received the B.Sc. degree in Electrical Engineering from National University of Sciences and Technology, Pakistan in 2006, M.Sc degree from University of Surrey, UK in 2009 and Ph.D. degree from COMSATS University Islamabad, Pakistan in 2018. He is currently a Research Fellow at the Centre for Vision, Speech and Signal Processing (CVSSP) at the University of Surrey, U.K. His research interests include Deep Learning, Machine Learning and Bayesian Signal Processing.

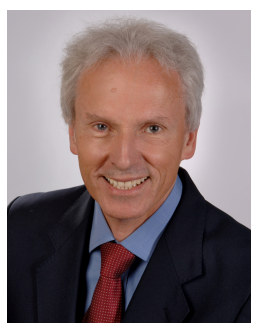

Josef Kittler (M'74-LM'12) received the B.A., Ph.D., and D.Sc. degrees from the University of Cambridge, in 1971, 1974, and 1991, respectively. $\mathrm{He}$ is a distinguished Professor of Machine Intelligence at the Centre for Vision, Speech and Signal Processing, University of Surrey, Guildford, U.K. He conducts research in biometrics, video and image dataset retrieval, medical image analysis, and cognitive vision. He published the textbook Pattern Recognition: A Statistical Approach and over 700 scientific papers. His publications have been cited more than 68,000 times (Google Scholar).

$\mathrm{He}$ is series editor of Springer Lecture Notes on Computer Science. He currently serves on the Editorial Boards of Pattern Recognition Letters, Pattern Recognition and Artificial Intelligence, Pattern Analysis and Applications. He also served as a member of the Editorial Board of IEEE Transactions on Pattern Analysis and Machine Intelligence during 1982-1985. He served on the Governing Board of the International Association for Pattern Recognition (IAPR) as one of the two British representatives during the period 1982-2005, President of the IAPR during 1994-1996. Currently he is a member of the KS Fu Prize Committee of IAPR. 